\title{
Finite-Time Distributed Energy-to-Peak Control for Uncertain Multiagent Systems
}

\author{
Qu Chenggang, ${ }^{1}$ Cao Xibin, ${ }^{1}$ Hamid Reza Karimi, ${ }^{2}$ Zhang Zhuo, ${ }^{3}$ and Zhang Zexu ${ }^{3}$ \\ ${ }^{1}$ Research Institute of Satellite Technology, Harbin Institute of Technology, Harbin 150080, China \\ ${ }^{2}$ Department of Engineering, Faculty of Engineering and Science, University of Agder, 4898 Grimstad, Norway \\ ${ }^{3}$ Deep Space Exploration Research Center, Harbin Institute of Technology, Harbin 150080, China \\ Correspondence should be addressed to Zhang Zexu; zexuzhang@hit.edu.cn
}

Received 21 November 2013; Accepted 4 December 2013; Published 9 January 2014

Academic Editor: Hui Zhang

Copyright (C) 2014 Qu Chenggang et al. This is an open access article distributed under the Creative Commons Attribution License, which permits unrestricted use, distribution, and reproduction in any medium, provided the original work is properly cited.

\begin{abstract}
This paper investigates the finite-time distributed $L_{2}-L_{\infty}$ consensus control problem of multiagent systems with parameter uncertainties. The relative states of neighboring agents are used to construct the control law and some agents know their own states. By substituting the control input into multiagent systems, an augmented closed-loop system is obtained. Then, we analyze its finite-time boundedness (FTB) and finite-time $L_{2}-L_{\infty}$ performance. A sufficient condition for the existence of the designed controller is given with the form of linear matrix inequalities (LMIs). Finally, simulation results are described.
\end{abstract}

\section{Introduction}

The coordination control problems of multiagent systems have attracted increasing attentions from various fields, such as formation flights $[1,2]$, multiple robots formation control [3, 4], air traffic control [5], and multivehicle systems cooperative control $[6,7]$. For the reason that centralized control is too expensive or even infeasible to accomplish, the distributed control protocol has been studied extensively in recent years.

The consensus problem for multiagent systems with switching communication topologies is studied in $[8,9]$, heterogeneous multiple agents are researched in [10], the distributed control problem for high-order multiagent systems is investigated in [11], the nonlinear uncertain multiagent systems are studied in [12], networked control problem for multiple agents with a leader is described in [13], multiagent systems with input time delays are investigated in [14], and, for the case that state information cannot be measured, a distributed output-feedback control for multiagent systems with Markov jumping is studied in [15]. However, to the best knowledge of the authors, there is no article investigating the finite-time energy-to-peak consensus problem for multiagent systems with parameter uncertainties.
For the reason that the forms of external disturbances are not exactly known and the existence of parameter uncertainties in systems, the robust control method is proposed. There are mainly three robust control theories. The first one is energy-to-energy control, which is the well-known $H_{\infty}$ control [16-19], where the external disturbance can be any form but energy bounded. The second theory is peak-to-peak control, in which worse cases of performance variables are required to be minimized under the peak bounded external disturbances; it can be seen that the conservatism of peakto-peak control is much less than the $H_{\infty}$ control. The last method is the energy-to-peak one, which is also called $l_{2}-l_{\infty}$ in discrete-time and $L_{2}-L_{\infty}$ in continuous-time systems. In energy-to-peak control, the external disturbances are energy bounded and the peak values of performance variables are required to be minimized, which is less conservative than the $H_{\infty}$ control. The energy-to-peak problem for Markovian jump systems is investigated in [20], the robust energyto-peak FIR equalization for time-varying communication channels is described in [21], and energy-to-peak filtering is designed in $[22,23]$.

In practical engineering, the main concern is the behavior of the system in a fixed finite-time interval. In such case, the finite-time stability (FTS) is introduced. A system is said 
to be finite-time stable if, in a given time interval, the state values will not exceed a certain upper bound under the initial conditions. The FTS method is applied to restrain the large state values in the initial period of time caused by the large initial conditions.

The FTS problem for switched systems is studied in [24], input-output FTS method is proposed in [25], and a timevarying system FTS problem is analyzed in [26]; the FTS is investigated for the singular impulsive systems in [27]. See for more details the references of the aforementioned literatures.

In this paper, we investigate the finite-time $L_{2}-L_{\infty}$ control problem for multiagent systems with parameter uncertainties. The rest of this paper is organized as follows. Some preliminaries on graph theory and problem formulation are given in Section 2. The finite-time distributed $L_{2}-L_{\infty}$ consensus control problem for multiagent systems with parameter uncertainties is investigated in Section 3. Numerical simulations are shown in Section 4. Section 5 concludes the paper.

In this paper, the following notations will be used: $R^{n \times n}$ denotes the set of $n \times n$ real matrix. The superscripts -1 and $T$ mean the inverse and the transpose of a matrix, respectively. The real matrix $P>0$ stands for the fact that $P$ is positive definite. $\lambda_{\text {min }}(P)$ and $\lambda_{\text {max }}(P)$, respectively, refer to the minimum and maximum eigenvalues of the matrix $P$. $\operatorname{diag}\left\{A_{1}, \ldots, A_{N}\right\}$ is a block-diagonal matrix. $A \otimes B$ is the Kronecker product of matrices $A$ and $B$. Further, some algorithms of Kronecker product applied are described as follows:

$$
\begin{gathered}
(A \otimes B)(C \otimes D)=(A C) \otimes(B D), \\
\left(A \otimes I_{n}\right)\left(I_{m} \otimes B\right)=\left(I_{m} \otimes B\right)\left(A \otimes I_{n}\right), \\
(A \otimes B)^{T}=A^{T} \otimes B^{T}, \quad(A \otimes B)^{-1}=A^{-1} \otimes B^{-1} .
\end{gathered}
$$

\section{Preliminaries}

2.1. Graph Theory. An undirected graph $\mathscr{G}=(\mathscr{V}, \mathscr{E})$ is a pair $(\mathscr{V}, \mathscr{E})$, where $\mathscr{V}=\left\{v_{1}, v_{2}, \ldots, v_{N}\right\}$ is the set of nodes and $\mathscr{E} \subseteq \mathscr{V} \times \mathscr{V}$ is the set of pairs of nodes, called edges. Two nodes $v_{i}$ and $v_{j}$ are called neighbouring agents, if $\left(v_{i}, v_{j}\right)$ is an edge of graph $\mathscr{G}$. A path on $\mathscr{G}_{f}$ from node $v_{i}$ to node $v_{j}$ is a sequence of edges $\left(v_{i}, v_{k_{1}}\right),\left(v_{k_{1}}, v_{k_{2}}\right), \ldots,\left(v_{k_{l}}, v_{j}\right)$. A graph is called connected graph if there exists a path from $v_{i}$ to $v_{j}$ for all pairs of $\left(v_{i}, v_{j}\right)$.

The adjacency matrix $\mathscr{A}=\left[a_{i j}\right] \in R^{N \times N}$ associated with undirected graph $\mathscr{G}$ is defined by $a_{i i}=0$ and $a_{i j}=a_{j i}=1$ if $\left(v_{i}, v_{j}\right) \in \mathscr{E}$ and $a_{i j}=a_{j i}=0$ otherwise. The Laplacian matrix $\mathscr{L}=\left[l_{i j}\right] \in R^{N \times N}$ is defined as $l_{i i}=\sum_{j=1}^{N} a_{i j}$ and $l_{i j}=-a_{i j}$ for $i \neq j$.

Define a diagonal matrix $\mathscr{D}=\operatorname{diag}\left\{d_{1}, \ldots, d_{N}\right\} \in R^{N \times N}$ : $d_{i}=1$ if the $i$ th agent knows its own state and 0 otherwise. Define a new matrix called modified Laplacian matrix $\widehat{\mathscr{L}}=$ $\mathscr{L}+\mathscr{D}$.

Assumption 1. The undirected topology graph $\mathscr{G}$ is connected and at least one agent is able to achieve its own state.
Lemma 2 (see [28]). If the graph $\mathscr{G}$ satisfies Assumption 1, then matrix $\widehat{\mathscr{L}}=\mathscr{L}+\mathscr{D}$ is positive definite.

2.2. Problem Formulation. Consider the following $N$ linear multiagent systems with parameter uncertainties:

$$
\begin{array}{r}
\dot{x}_{i}=\left(A+\Delta A_{i}\right) x_{i}+B u_{i}+D w_{i}, \quad z_{i}=x_{i}, \\
i=1, \ldots, N,
\end{array}
$$

where the subscripts $i$ denote the $i$ th agent. $x_{i} \in R^{n}$ stands for the state variable, $u_{i} \in R^{p}$ is the control input, $w_{i} \in R^{m}$ means the external disturbance, and $z_{i} \in R^{n}$ refers to the required performance variable. $A, B$, and $D$ are constant matrices with the compatible dimensions; matrix $\Delta A_{i}$ denotes the parameter uncertainty which is unknown and described as $\Delta A_{i}=E \Sigma_{i}(t) F$, where $\Sigma_{i}(t)$ stands for the time-varying uncertainty and satisfies the following condition:

$$
\Sigma_{i}^{T}(t) \Sigma_{i}(t) \leq \delta^{2} I \quad i=1, \ldots, N
$$

with the constant parameter $\delta>0$, where $E$ and $F$ are constant matrices which are used to characterize the structure of the uncertainty.

Therefore, it is called that the multi-agent systems have the same nominal dynamics which are the certain parts, but the different uncertainties with each other.

Similar to $[28,29]$, in this paper, we aim to make multiple agents achieve the consensus and their states converge to zero thus, the controller is described as

$$
u_{i}=c K\left[\sum_{j=1}^{N} a_{i j}\left(x_{i}-x_{j}\right)+d_{i} x_{i}\right], \quad i=1, \ldots, N,
$$

where the scalar $c>0$ denotes the coupling strength, $K$ stands for the relative state-feedback control gain matrix to be determined, $a_{i j}, i, j \in\{1, \ldots, N\}$, is the element of adjacency matrix $\mathscr{A}$ associated with the communication topology graph, and $\mathscr{G}, d_{i}$ are constant scalars which satisfy $d_{i}=1, i=1, \ldots, q$, and $d_{i}=0, i=q+1, \ldots, N$; that is to say, agents $1, \ldots, q$ are able to achieve their own states, but agents $q+1, \ldots, N$ cannot get their own states.

We define the following augmented variables:

$$
x=\left[\begin{array}{c}
x_{1} \\
\vdots \\
x_{N}
\end{array}\right], \quad w=\left[\begin{array}{c}
w_{1} \\
\vdots \\
w_{N}
\end{array}\right], \quad z=\left[\begin{array}{c}
z_{1} \\
\vdots \\
z_{N}
\end{array}\right] .
$$

Then, the closed-loop system consisting of (2) and (4) can be rewritten as

$$
\begin{gathered}
\dot{x}=\left[I_{N} \otimes A+c \widehat{L} \otimes B K+\left(I_{N} \otimes E\right) \Sigma\left(I_{N} \otimes F\right)\right] x \\
+\left(I_{N} \otimes D\right) w, \quad \\
z=x,
\end{gathered}
$$

where $\widehat{\mathscr{L}}=\mathscr{L}+\mathscr{D}$ and $\Sigma=\operatorname{diag}\left\{\Sigma_{1}, \ldots, \Sigma_{N}\right\}$. It is obvious that matrix $\widehat{\mathscr{L}}$ is positive definite based on Assumption 1 and Lemma 2. 
In order to analyze the finite-time $L_{2}-L_{\infty}$ performance of the closed-loop system (6) with uncertainties, some definitions and a lemma are described as follows.

Definition 3. The finite-time energy-to-peak gain of the transfer function matrix is described by

$$
\Gamma_{\mathrm{ep}(w z)}=\sqrt{\frac{\sup _{t \in(0, T]}\left[z^{T}(t) z(t)\right]}{\int_{0}^{T} w^{T}(t) w(t) d t}} .
$$

Definition 4 (finite-time boundedness (FTB) [30]). A system is called FTB with respect to $\left(c_{1}, c_{2}, \bar{\omega}, T\right)$, where $0<c_{1}<c_{2}$, if the state variables satisfy

$$
x^{T}(t) x(t) \leq c_{2}, \quad \forall t \in(0, T]
$$

with the conditions

$$
x^{T}(0) x(0) \leq c_{1}, \quad \int_{0}^{T} w^{T}(t) w(t) d t \leq \bar{\omega} .
$$

Definition 5. The closed-loop system (6) is said to be FTB with a finite-time $L_{2}-L_{\infty}$ performance $\gamma$, if the following conditions hold.

(1) System (6) is FTB with respect to $\left(c_{1}, c_{2}, \bar{\omega}, T\right)$.

(2) Under zero initial conditions, the $L_{2}-L_{\infty}$ performance satisfies the following condition:

$$
\sup _{t \in(0, T]}\left[z^{T}(t) z(t)\right]<\gamma^{2} \int_{0}^{T} w^{T}(t) w(t) d t .
$$

That is, $\Gamma_{\mathrm{ep}(w z)}<\gamma$.

In addition, based on Definition 3, it is derived from [31] that

$$
\Gamma_{\mathrm{ep}(w z)}=\frac{1}{2 \pi} \lambda_{\max }\left(\int_{0}^{T} T_{w z}(j \omega) T_{w z}^{T}(j \omega) d \omega\right),
$$

where $T_{w z}$ is the transfer function matrix of closed-loop system (6); note that the right side of (11) is the finite-time $\mathrm{H}_{2}$ norm of the transfer function matrix.

Lemma 6 (see [32]). Let $\Theta=\Theta^{T}$; let $\bar{E}$ and $\bar{F}$ be real matrices with appropriate dimensions; $\Sigma(k)$ satisfies $\Sigma^{T}(k) \Sigma(k) \leq I$. Then the following condition:

$$
\Theta+\bar{E} \Sigma(k) \bar{F}+(\bar{E} \Sigma(k) \bar{F})^{T}<0,
$$

holds if there exists a positive scalar $\varepsilon>0$ such that the following inequality is satisfied:

$$
\left[\begin{array}{ccc}
\Theta & \varepsilon \bar{E} & \bar{F}^{T} \\
* & -\varepsilon I & 0 \\
* & * & -\varepsilon I
\end{array}\right]<0 .
$$

Theorem 7. For a given scalar $\gamma>0$, system (6) is FTB with the finite-time $L_{2}-L_{\infty}$ performance $\gamma$, if and only if the following $N$ systems are finite-time bounded and the finite-time $L_{2}-L_{\infty}$ norm of their transfer function matrices $\Gamma_{e p\left(\widehat{w}_{i} \widehat{z}_{i}\right)}<\gamma$ :

$$
\begin{array}{r}
\dot{\hat{x}}_{i}=\left(A+\Delta A_{i}+c \lambda_{i} B K\right) \widehat{x}_{i}+D \widehat{w}_{i}, \quad \widehat{z}_{i}=\widehat{x}_{i}, \\
i=1, \ldots, N,
\end{array}
$$

where $\lambda_{i}$ is the ith eigenvalue of $\widehat{\mathscr{L}}$.

Proof. Let $U \in R^{N \times N}$ be a unitary matrix such that $U^{T} \widehat{\mathscr{L}} U=$ $\Lambda=\operatorname{diag}\left\{\lambda_{1}, \ldots, \lambda_{N}\right\}$. Define new variables $\hat{x}=\left(U^{T} \otimes I_{n}\right) x$, $\widehat{w}=\left(U^{T} \otimes I_{m}\right) w$, and $\widehat{z}=\left(U^{T} \otimes I_{n}\right) z$, where

$$
\widehat{x}=\left[\begin{array}{c}
\widehat{x}_{1} \\
\vdots \\
\widehat{x}_{N}
\end{array}\right], \quad \widehat{w}=\left[\begin{array}{c}
\widehat{w}_{1} \\
\vdots \\
\widehat{w}_{N}
\end{array}\right], \quad \widehat{z}=\left[\begin{array}{c}
\widehat{z}_{1} \\
\vdots \\
\widehat{z}_{N}
\end{array}\right] .
$$

Therefore, we have

$$
\begin{gathered}
\dot{\hat{x}}=\left(U^{T} \otimes I_{n}\right)\left[I_{N} \otimes A+c \widehat{\mathscr{L}} \otimes B K+\left(I_{N} \otimes E\right) \Sigma\left(I_{N} \otimes F\right)\right] \\
\times\left(U \otimes I_{n}\right) \widehat{x}+\left(U^{T} \otimes I_{n}\right)\left(I_{N} \otimes D\right)\left(U \otimes I_{m}\right) \widehat{w} \\
=\left[I_{N} \otimes A+c \Lambda \otimes B K+\left(U^{T} \otimes I_{n}\right)\left(I_{N} \otimes E \Sigma_{i} F\right)\right. \\
\left.\times\left(U \otimes I_{n}\right)\right] \widehat{x}+\left(I_{N} \otimes D\right) \widehat{w} \\
=\left[I_{N} \otimes A+c \Lambda \otimes B K+\left(I_{N} \otimes E \Sigma_{i} F\right)\right] \widehat{x}+\left(I_{N} \otimes D\right) \widehat{w} .
\end{gathered}
$$

Hence, the closed-loop system (6) can be regarded as

$$
\begin{gathered}
\dot{\hat{x}}=\left[I_{N} \otimes A+c \Lambda \otimes B K+\left(I_{N} \otimes E \Sigma_{i} F\right)\right] \widehat{x}+\left(I_{N} \otimes D\right) \widehat{w}, \\
\widehat{z}=\widehat{x} .
\end{gathered}
$$

Note that the system in (17) is composed of the $N$ systems in (14).

It can be seen that the system (17) is obtained by applying the elementary transformation to system (6). Thus the finitetime stability (boundedness) of systems (6) and (17) is equivalent.

It is concluded from (6), (14), and (17) that

$$
T_{\widehat{w} \bar{z}}=\operatorname{diag}\left\{T_{\widehat{w}_{1} \widehat{z}_{1}}, \ldots, T_{\widehat{w}_{N} \widehat{z}_{N}}\right\}=\left(U^{T} \otimes I_{m}\right) T_{w z}\left(U^{T} \otimes I_{n}\right) .
$$

Via the finite-time $L_{2}-L_{\infty}$ norm described in (11), it implies that

$$
\Gamma_{\mathrm{ep}(w z)}=\Gamma_{\mathrm{ep}(\widehat{w} \hat{z})}=\max _{i=1, \ldots, N} \Gamma_{\mathrm{ep}\left(\widehat{w}_{i} \bar{z}_{i}\right)} .
$$

Therefore, it is obvious that $\Gamma_{\mathrm{ep}(w z)}<\gamma$ if and only if $\Gamma_{\mathrm{ep}\left(\widehat{w}_{i} \widehat{z}_{i}\right)}<$ $\gamma, i \in\{1, \ldots, N\}$. This completes the proof. 
Remark 8. It is shown in Theorem 7 that the distributed finite-time energy-to-peak control problem of system (6) is equivalent to the finite-time energy-to-peak control problems of a set of decoupled linear systems having the same dimensions as a single agent with the assumption that the nominal dynamics of agents are identical.

In the next section, we are going to derive the sufficient condition for the existence of the control input and give the expression of feedback gain matrix $K$. Select the coupling strength $c \geq c_{\text {th }}$, where

$$
c_{\mathrm{th}}=\frac{\tau}{\min _{i=1, \ldots, N}\left(\lambda_{i}\right)}
$$

\section{Finite-Time Energy-to-Peak Control}

Theorem 9. The closed-loop network in (6) with a distributed controller described in (4) is finite-time bounded with a finitetime energy-to-peak performance $\gamma$, if there exist positive definite symmetric matrix $Q$, scalars $\tau>0, \alpha>0$, and $\varepsilon>0$ such that

$$
\begin{aligned}
& {\left[\begin{array}{ccccc}
-\alpha Q+A Q+Q A^{T}-\tau B B^{T} & D & \mathbb{Q} & \varepsilon E & \delta Q F^{T} \\
D^{T} & -\gamma^{2} I & 0 & 0 & 0 \\
Q & 0 & -I & 0 & 0 \\
\varepsilon E^{T} & 0 & 0 & -\varepsilon I & 0 \\
\delta Q F & 0 & 0 & 0 & -\varepsilon I
\end{array}\right]<0} \\
& {\left[\begin{array}{cc}
-e^{-\alpha T} Q & \mathcal{Q} \\
\mathcal{Q} & -I
\end{array}\right]<0 .}
\end{aligned}
$$

In addition, the feedback gain matrix is $K=-(1 / 2) B^{T} Q^{-1}$.

Proof. Based on the Schur Complement Lemma, inequality (21) can be regarded as

$$
\begin{aligned}
-\alpha \mathscr{Q} & +A \mathscr{Q}+\mathscr{Q} A^{T}-\tau B B^{T}+\gamma^{-2} D D^{T}+Q^{2}+\varepsilon E E^{T} \\
& +\varepsilon^{-1} \delta^{2} Q F^{T} F Q<0 .
\end{aligned}
$$

Thus, by using (20), we have

$$
\begin{aligned}
-\alpha Q & +A Q+Q A^{T}-c \lambda_{i} B B^{T}+\gamma^{-2} D D^{T}+Q^{2}+\varepsilon E E^{T} \\
& +\varepsilon^{-1} \delta^{2} Q F^{T} F Q<0 .
\end{aligned}
$$

We choose the control gain matrix as $K=-(1 / 2) B^{T} Q^{-1}$; then we can obtain

$$
\begin{aligned}
-\alpha Q & +A Q+Q A^{T}+c \lambda_{i} Q K^{T} B^{T}+c \lambda_{i} B K Q+\gamma^{-2} D D^{T} \\
& +Q^{2}+\varepsilon E E^{T}+\varepsilon^{-1} \delta^{2} Q F^{T} F Q<0 .
\end{aligned}
$$

By using Schur Complement Lemma again, the above inequality is equivalent to

$$
\left[\begin{array}{ccccc}
\Omega_{11} & D & \mathcal{Q} & \varepsilon E & \delta Q F^{T} \\
D^{T} & -\gamma^{2} I & 0 & 0 & 0 \\
Q & 0 & -I & 0 & 0 \\
\varepsilon E^{T} & 0 & 0 & -\varepsilon I & 0 \\
\delta Q F & 0 & 0 & 0 & -\varepsilon I
\end{array}\right]<0,
$$

where

$$
\Omega_{11}=-\alpha Q+Q\left(A+c \lambda_{i} B K\right)^{T}+\left(A+c \lambda_{i} B K\right) Q .
$$

By applying Lemma 6, we can obtain the following inequality if the inequality (26) holds:

$$
\left[\begin{array}{ccc}
\Pi_{11} & D & \mathbb{Q} \\
D^{T} & -\gamma^{2} I & 0 \\
\mathcal{Q} & 0 & -I
\end{array}\right]<0,
$$

where

$$
\begin{aligned}
\Pi_{11}= & -\alpha \mathscr{Q}+\mathscr{Q}\left(A+c \lambda_{i} B K+E \Sigma_{i} F\right)^{T} \\
& +\left(A+c \lambda_{i} B K E \Sigma_{i} F\right) Q
\end{aligned}
$$

By multiplying the left and right sides of inequality (28) by $\operatorname{diag}\left\{Q^{-1}, I, I\right\}$, we have

$$
\left[\begin{array}{ccc}
\widetilde{\Pi}_{11} & \mathcal{Q}^{-1} D & I \\
D^{T} Q^{-1} & -\gamma^{2} I & 0 \\
I & 0 & -I
\end{array}\right]<0,
$$

where

$$
\begin{aligned}
\widetilde{\Pi}_{11}= & -\alpha \mathscr{Q}^{-1}+\left(A+c \lambda_{i} B K+E \Sigma_{i} F\right)^{T} \mathscr{Q}^{-1} \\
& +\mathscr{Q}^{-1}\left(A+c \lambda_{i} B K E \Sigma_{i} F\right) .
\end{aligned}
$$

Let $P=Q^{-1}$; we can get

$$
\left[\begin{array}{ccc}
\bar{\Pi}_{11} & P D & I \\
D^{T} P & -\gamma^{2} I & 0 \\
I & 0 & -I
\end{array}\right]<0,
$$

where

$$
\begin{aligned}
\bar{\Pi}_{11}= & -\alpha P+\left(A+c \lambda_{i} B K+E \Sigma_{i} F\right)^{T} P \\
& +P\left(A+c \lambda_{i} B K E \Sigma_{i} F\right) .
\end{aligned}
$$

It is obvious that $P$ is also a positive definite symmetric matrix. By using Schur Complement Lemma, inequality (32) is equivalent to

$$
\left[\begin{array}{cc}
\Xi_{11} & P D \\
D^{T} P & -\gamma^{2} I
\end{array}\right]<0,
$$


where

$$
\begin{aligned}
\Xi_{11}= & -\alpha P+I+\left(A+c \lambda_{i} B K+E \Sigma_{i} F\right)^{T} P \\
& +P\left(A+c \lambda_{i} B K E \Sigma_{i} F\right) .
\end{aligned}
$$

We choose the Lyapunov function for the $N$ systems in (14) as follows:

$$
V_{i}(t)=\widehat{x}_{i}^{T}(t) P \widehat{x}_{i}(t), \quad i=1, \ldots, N
$$

The derivate of the aforementioned Lyap unov function is

$$
\begin{aligned}
\dot{V}_{i}= & \dot{\hat{x}}_{i}^{T} P \widehat{x}_{i}+\widehat{x}_{i}^{T} P \dot{\hat{x}}_{i} \\
= & \widehat{x}_{i}^{T}\left[\left(A+c \lambda_{i} B K+E \Sigma_{i} F\right)^{T} P+P\left(A+c \lambda_{i} B K+E \Sigma_{i} F\right)\right] \\
& \times \widehat{x}_{i}+\widehat{x}_{i}^{T} P D \widehat{w}_{i}+\widehat{w}_{i}^{T} D^{T} P \widehat{x}_{i} .
\end{aligned}
$$

Therefore, it is derived from the inequality (34) that

$$
\dot{V}_{i}+\widehat{x}_{i}^{T} \widehat{x}_{i}-\alpha \widehat{x}_{i}^{T} P \widehat{x}_{i}-\gamma^{2} \widehat{w}_{i}^{T} \widehat{w}_{i}<0 .
$$

That is,

$$
\dot{V}_{i}<\alpha V_{i}+\gamma^{2} \widehat{w}_{i}^{T} \widehat{w}_{i}-\widehat{z}_{i}^{T} \widehat{z}_{i}
$$

Then we have

$$
\dot{V}_{i}<\alpha V_{i}+\gamma^{2} \widehat{w}_{i}^{T} \widehat{w}_{i}
$$

Hence, the above inequality can be regarded as

$$
\frac{d}{d t}\left[e^{-\alpha t} V_{i}(t)\right]<e^{-\alpha t} \gamma^{2} \widehat{w}_{i}^{T}(t) \widehat{w}_{i}(t) .
$$

By integrating the aforementioned inequality from 0 to $t$, we can obtain

$$
\begin{aligned}
e^{-\alpha t} V_{i}(t) & <V_{i}(0)+\gamma^{2} \int_{0}^{t} e^{-\alpha s} \widehat{w}_{i}^{T}(s) \widehat{w}_{i}(s) d s \\
& \leq V_{i}(0)+\gamma^{2} \int_{0}^{t} \widehat{w}_{i}^{T}(s) \widehat{w}_{i}(s) d s \\
& \leq \widehat{x}_{i}^{T}(0) P \widehat{x}_{i}(0)+\gamma^{2} \int_{0}^{T} \widehat{w}_{i}^{T}(t) \widehat{w}_{i}(t) d t \\
& =\lambda_{\max }(P) \widehat{x}_{i}^{T}(0) \widehat{x}_{i}(0)+\gamma^{2} \int_{0}^{T} \widehat{w}_{i}^{T}(t) \widehat{w}_{i}(t) d t
\end{aligned}
$$

For the initial conditions $\widehat{x}_{i}^{T}(0) \widehat{x}_{i}(0) \leq c_{1}$ and finite-time energy-bounded disturbance conditions $\int_{0}^{T} \widehat{w}_{i}^{T}(t) \widehat{w}_{i}(t) d t \leq$ $\bar{\omega}$ hold, the above inequality can be rewritten as

$$
V_{i}(t)<e^{\alpha t} c_{1} \lambda_{\max }(P)+\gamma^{2} \bar{\omega} \leq e^{\alpha T} c_{1} \lambda_{\max }(P)+\gamma^{2} \bar{\omega} .
$$

For the reason that $V_{i}(t) \geq \lambda_{\min }(P) \widehat{x}_{i}^{T}(t) \widehat{x}_{i}(t)$, it concludes that

$$
\widehat{x}_{i}^{T}(t) \widehat{x}_{i}(t)<\frac{e^{\alpha T} c_{1} \lambda_{\max }(P)+\gamma^{2} \bar{\omega}}{\lambda_{\min }(P)}=c_{2}, \quad t \in(0, T] .
$$

Therefore, the $N$ systems with parameter uncertainties in (14) are all finite-time bounded with respect to $\left(c_{1}, c_{2}, \bar{\omega}, T\right)$.

Next, we are going to prove the finite-time $L_{2}-L_{\infty}$ performance of $N$ systems (14) which contain parameter uncertainties. Multiplying the left and right sides of inequality (22) by $\operatorname{diag}\left\{Q^{-1}, I\right\}$ gives

$$
\left[\begin{array}{cc}
-e^{-\alpha T} Q^{-1} & I \\
I & -I
\end{array}\right]<0,
$$

where $\mathbb{Q}^{-1}=P$; then we have

$$
\left[\begin{array}{cc}
-e^{-\alpha T} P & I \\
I & -I
\end{array}\right]<0
$$

By using Schur Complement Lemma, the inequality (46) is equivalent to

$$
I<e^{-\alpha T} P \text {. }
$$

Therefore, we have

$$
\widehat{z}_{i}^{T}(t) \widehat{z}_{i}(t)=\widehat{x}_{i}^{T}(t) \widehat{x}_{i}(t)<e^{-\alpha T} \widehat{x}_{i}^{T}(t) P \widehat{x}_{i}(t)=e^{-\alpha T} V_{i}(t) .
$$

By integrating the inequality (41) from 0 to $t$, we have

$$
e^{-\alpha t} V_{i}(t)<V_{i}(0)+\gamma^{2} \int_{0}^{t} e^{-\alpha s} \widehat{w}_{i}^{T}(s) \widehat{w}_{i}(s) d s .
$$

Under the zero initial condition, which means that $V_{i}(0)=0$, we will have

$$
\begin{aligned}
e^{-\alpha t} V_{i}(t) & <\gamma^{2} \int_{0}^{t} e^{-\alpha s} \widehat{w}_{i}^{T}(s) \widehat{w}_{i}(s) d s \\
& \leq \gamma^{2} \int_{0}^{T} e^{-\alpha t} \widehat{w}_{i}^{T}(t) \widehat{w}_{i}(t) d t \\
& \leq \gamma^{2} \int_{0}^{T} \widehat{w}_{i}^{T}(t) \widehat{w}_{i}(t) d t .
\end{aligned}
$$

Therefore, by combing the inequalities (48) and (50), we can obtain

$$
\begin{aligned}
\widehat{z}_{i}^{T}(t) \widehat{z}_{i}(t) & <e^{-\alpha T} V_{i}(t) \leq e^{-\alpha t} V_{i}(t) \\
& <\gamma^{2} \int_{0}^{T} \widehat{w}_{i}^{T}(t) \widehat{w}_{i}(t) d t \quad \forall t \in(0, T] .
\end{aligned}
$$

Hence, we have

$$
\sup _{t \in(0, T]}\left[\widehat{z}_{i}^{T}(t) \widehat{z}_{i}(t)\right]<\gamma^{2} \int_{0}^{T} \widehat{w}_{i}^{T}(t) \widehat{w}_{i}(t) d t .
$$

Therefore, the $N$ systems (14) are all finite-time bounded with a finite-time $L_{2}-L_{\infty}$ performance $\gamma$. Further, based on Theorem 7 the closed-loop network with parameter uncertainties in (6) is FTB with a finite-time $L_{2}-L_{\infty}$ performance $\gamma$, if and only if the systems (14) are all FTB with a finite-time $L_{2}-L_{\infty}$ performance $\gamma$.

Hence, system (6) is FTB with a finite-time $L_{2}-L_{\infty}$ performance $\gamma$ under the control inputs (4), if the inequalities (21) and (22) hold. This completes the proof. 
Corollary 10. By applying Theorems 7 and 9, it can be seen that the minimum finite-time $L_{2}-L_{\infty}$ performance index $\gamma_{\min }$ can be obtained by the following optimization problem:

\section{minimize $\gamma$}

subject to LMIs (21) and (22), with $Q>0, \tau>0, \gamma>0$, $\alpha>0, \varepsilon>0$.

Remark 11. The above designs a distributed energy-to-peak controller which is decoupled from the communication topologies. Here, we only need to select the coupling strength $c$ to be large enough to make the controller can be used to multiple connected communication topology graphs.

\section{Numerical Simulations}

In this section, simulation examples are provided to illustrate the effectiveness of the theoretical results. Consider that the multiagent systems with parameter uncertainties consist of six agents as follows:

$$
\begin{array}{r}
\dot{x}_{i}=\left(A+\Delta A_{i}\right) x_{i}+B u_{i}+D w_{i}, \quad z_{i}=x_{i}, \\
i=1, \ldots, 6,
\end{array}
$$

where $\Delta A_{i}=E \Sigma_{i}(t) F, \Sigma_{i}^{T}(t) \Sigma_{i}(t) \leq \delta^{2} I$, and

$$
\begin{gathered}
A=\left[\begin{array}{ll}
0 & 1 \\
0 & 0
\end{array}\right], \quad B=\left[\begin{array}{l}
0 \\
1
\end{array}\right], \quad D=\left[\begin{array}{cc}
0.1 & 0 \\
0 & 0.1
\end{array}\right], \\
E=\left[\begin{array}{l}
0 \\
1
\end{array}\right], \quad F=\left[\begin{array}{ll}
0 & 1
\end{array}\right], \quad \delta=0.2 .
\end{gathered}
$$

Hence, by applying Corollary 10 and choosing $\alpha=0.1$ and $T=2$, optimal solutions of LMIs (21) and (22) are calculated by

$$
\begin{gathered}
Q \mathcal{Q}=\left[\begin{array}{cc}
0.3887 & -0.2970 \\
-0.2970 & 0.5903
\end{array}\right], \\
\tau=3.0003 \\
\gamma_{\min }=0.6213 .
\end{gathered}
$$

Therefore, the corresponding matrix $P$ and the control gain matrix $K$ are, respectively, calculated by

$$
\begin{gathered}
P=Q^{-1}=\left[\begin{array}{ll}
4.1803 & 2.0134 \\
2.0134 & 2.7523
\end{array}\right], \\
K=-\frac{1}{2} B^{T} Q^{-1}=\left[\begin{array}{ll}
-1.0517 & -1.3762
\end{array}\right] .
\end{gathered}
$$

Figure 1 shows four different communication topology graphs with six agents, a loop, that is, an edge start from one agent and end to itself, means that the agent knows its own state. An edge from agent $i$ to agent $j$ means that agent $j$ can receive information from agent $i$. Different cases of network links are, respectively, shown in graphs $\mathscr{G}_{1}, \mathscr{G}_{2}$, and $\mathscr{G}_{3}$, which are subgraphs of $\mathscr{G}$.
Hence, the corresponding weighted adjacency matrices are described by

$$
\begin{aligned}
\mathscr{A}_{1}=\left[\begin{array}{llllll}
0 & 1 & 0 & 0 & 0 & 1 \\
1 & 0 & 1 & 0 & 0 & 0 \\
0 & 1 & 0 & 0 & 0 & 0 \\
0 & 0 & 0 & 0 & 1 & 0 \\
0 & 0 & 0 & 1 & 0 & 1 \\
1 & 0 & 0 & 0 & 1 & 0
\end{array}\right], & \mathscr{A}_{2}=\left[\begin{array}{llllll}
0 & 1 & 0 & 0 & 0 & 1 \\
1 & 0 & 0 & 0 & 0 & 0 \\
0 & 0 & 0 & 1 & 0 & 1 \\
0 & 0 & 1 & 0 & 1 & 0 \\
0 & 0 & 0 & 1 & 0 & 0 \\
1 & 0 & 1 & 0 & 0 & 0
\end{array}\right], \\
\mathscr{A}_{3}=\left[\begin{array}{llllll}
0 & 1 & 0 & 1 & 1 & 1 \\
1 & 0 & 1 & 0 & 0 & 0 \\
0 & 1 & 0 & 1 & 0 & 0 \\
1 & 0 & 1 & 0 & 1 & 0 \\
1 & 0 & 0 & 1 & 0 & 0 \\
1 & 0 & 0 & 0 & 0 & 0
\end{array}\right], & \mathscr{A}=\left[\begin{array}{llllll}
0 & 1 & 1 & 1 & 1 & 1 \\
1 & 0 & 1 & 1 & 1 & 1 \\
1 & 1 & 0 & 1 & 1 & 1 \\
1 & 1 & 1 & 0 & 1 & 1 \\
1 & 1 & 1 & 1 & 0 & 1 \\
1 & 1 & 1 & 1 & 1 & 0
\end{array}\right] .
\end{aligned}
$$

Then the corresponding own-state matrices are

$$
\begin{array}{ll}
\mathscr{D}_{1}=\left[\begin{array}{llllll}
1 & 0 & 0 & 0 & 0 & 0 \\
0 & 0 & 0 & 0 & 0 & 0 \\
0 & 0 & 0 & 0 & 0 & 0 \\
0 & 0 & 0 & 0 & 0 & 0 \\
0 & 0 & 0 & 0 & 0 & 0 \\
0 & 0 & 0 & 0 & 0 & 0
\end{array}\right], & \mathscr{D}_{2}=\left[\begin{array}{llllll}
1 & 0 & 0 & 0 & 0 & 0 \\
0 & 1 & 0 & 0 & 0 & 0 \\
0 & 0 & 0 & 0 & 0 & 0 \\
0 & 0 & 0 & 0 & 0 & 0 \\
0 & 0 & 0 & 0 & 0 & 0 \\
0 & 0 & 0 & 0 & 0 & 0
\end{array}\right], \\
\mathscr{D}_{3}=\left[\begin{array}{llllll}
1 & 0 & 0 & 0 & 0 & 0 \\
0 & 1 & 0 & 0 & 0 & 0 \\
0 & 0 & 1 & 0 & 0 & 0 \\
0 & 0 & 0 & 0 & 0 & 0 \\
0 & 0 & 0 & 0 & 0 & 0 \\
0 & 0 & 0 & 0 & 0 & 0
\end{array}\right], & \mathscr{D}=\left[\begin{array}{llllll}
1 & 0 & 0 & 0 & 0 & 0 \\
0 & 1 & 0 & 0 & 0 & 0 \\
0 & 0 & 1 & 0 & 0 & 0 \\
0 & 0 & 0 & 1 & 0 & 0 \\
0 & 0 & 0 & 0 & 1 & 0 \\
0 & 0 & 0 & 0 & 0 & 1
\end{array}\right] .
\end{array}
$$

Then the corresponding modified Laplacian matrices are described by

$$
\begin{aligned}
& \widehat{\mathscr{L}}_{1}=\left[\begin{array}{cccccc}
3 & -1 & 0 & 0 & 0 & -1 \\
-1 & 2 & -1 & 0 & 0 & 0 \\
0 & -1 & 1 & 0 & 0 & 0 \\
0 & 0 & 0 & 1 & -1 & 0 \\
0 & 0 & 0 & -1 & 2 & -1 \\
-1 & 0 & 0 & 0 & -1 & 2
\end{array}\right], \\
& \widehat{\mathscr{L}}_{2}=\left[\begin{array}{cccccc}
3 & -1 & 0 & 0 & 0 & -1 \\
-1 & 2 & 0 & 0 & 0 & 0 \\
0 & 0 & 2 & -1 & 0 & -1 \\
0 & 0 & -1 & 2 & -1 & 0 \\
0 & 0 & 0 & -1 & 1 & 0 \\
-1 & 0 & -1 & 0 & 0 & 2
\end{array}\right], \\
& \widehat{\mathscr{L}}_{3}=\left[\begin{array}{cccccc}
5 & -1 & 0 & -1 & -1 & -1 \\
-1 & 3 & -1 & 0 & 0 & 0 \\
0 & -1 & 3 & -1 & 0 & 0 \\
-1 & 0 & -1 & 3 & -1 & 0 \\
-1 & 0 & 0 & -1 & 2 & 0 \\
-1 & 0 & 0 & 0 & 0 & 1
\end{array}\right],
\end{aligned}
$$




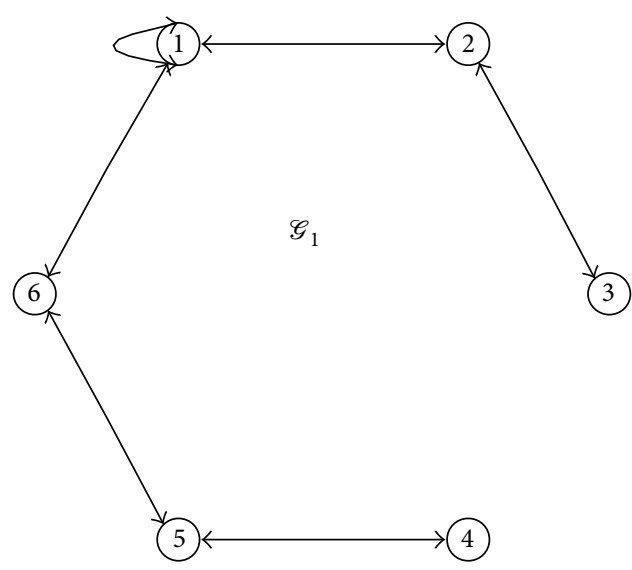

(a)

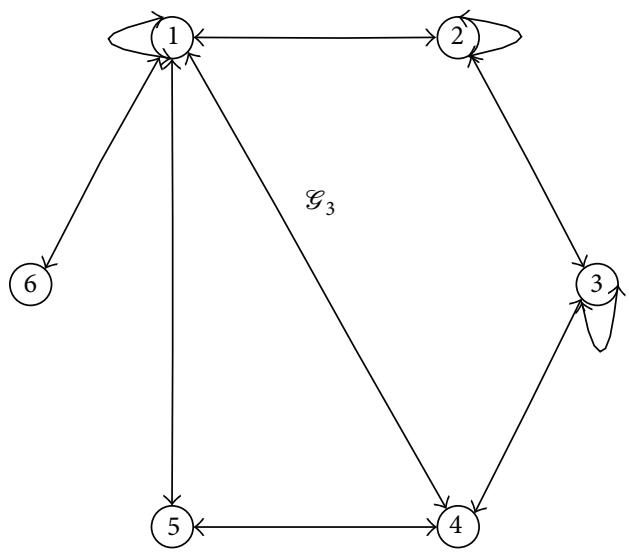

(c)

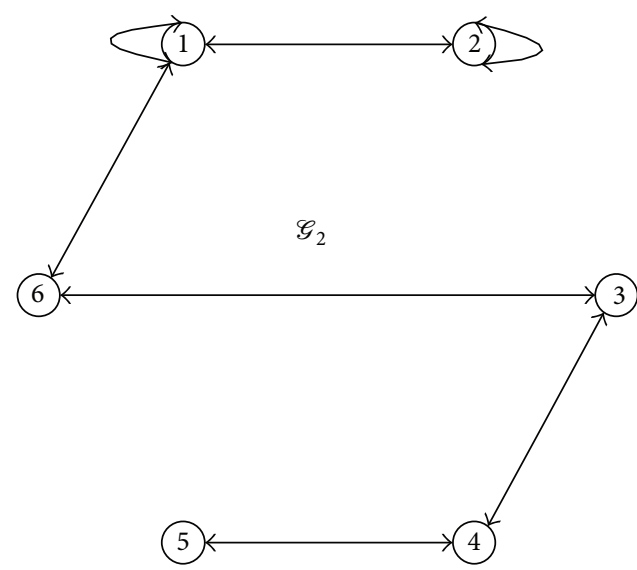

(b)

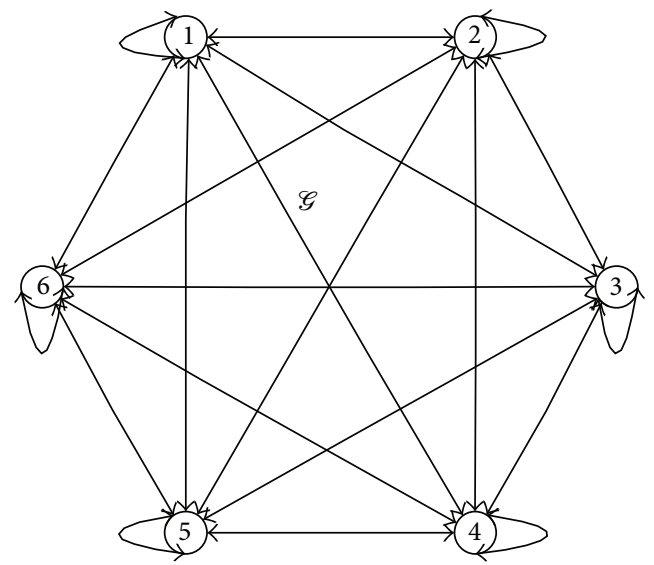

(d)

FIGURE 1: The communication topologies $\mathscr{G}_{1}, \mathscr{G}_{2}, \mathscr{G}_{3}$, and $\mathscr{G}$.

$$
\widehat{\mathscr{L}}=\left[\begin{array}{cccccc}
6 & -1 & -1 & -1 & -1 & -1 \\
-1 & 6 & -1 & -1 & -1 & -1 \\
-1 & -1 & 6 & -1 & -1 & -1 \\
-1 & -1 & -1 & 6 & -1 & -1 \\
-1 & -1 & -1 & -1 & 6 & -1 \\
-1 & -1 & -1 & -1 & -1 & 6
\end{array}\right]
$$

Hence, we can calculate the corresponding eigenvalues as follows:

$$
\begin{aligned}
& \lambda_{11}=0.1031, \quad \lambda_{12}=0.2903, \quad \lambda_{13}=1.2054, \\
& \mathscr{G}_{1}: \quad \lambda_{14}=2.1673, \quad \lambda_{15}=3.0735, \quad \lambda_{16}=4.1604 \text {, } \\
& \lambda_{21}=0.0913, \quad \lambda_{22}=0.7302, \quad \lambda_{23}=1.4413,
\end{aligned}
$$

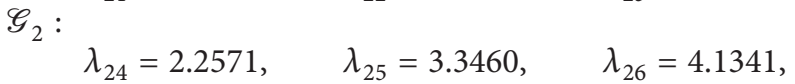

$$
\begin{aligned}
& \lambda_{31}=0.3650, \quad \lambda_{32}=1.0423, \quad \lambda_{33}=2.0000, \\
& \mathscr{G}_{3}: \\
& \lambda_{34}=3.2526, \quad \lambda_{35}=4.2366, \quad \lambda_{36}=6.1034,
\end{aligned}
$$

$$
\begin{aligned}
\lambda_{1}=1.0000, & \lambda_{2}=7.0000, & \lambda_{3}=7.0000, \\
\lambda_{4}=7.0000, & \lambda_{5}=7.0000, & \lambda_{6}=7.0000 .
\end{aligned}
$$

The corresponding minimum eigenvalues are

$$
\begin{array}{ll}
\lambda_{\min \left(\widehat{\mathscr{L}}_{1}\right)}=0.1031, & \lambda_{\min \left(\widehat{\mathscr{L}}_{2}\right)}=0.0913, \\
\lambda_{\min \left(\widehat{\mathscr{L}}_{3}\right)}=0.3650, & \lambda_{\min (\widehat{\mathscr{L}})}=1.0000 .
\end{array}
$$

Therefore, in order to make feedback control gain $c K$ be applied to the four different graphs, the scalar $c$ should be chosen large enough as

$$
\begin{aligned}
c \geq c_{\text {th }} & =\frac{\tau}{\min \left\{\lambda_{\min \left(\widehat{\mathscr{L}}_{1}\right)}, \lambda_{\min \left(\widehat{\mathscr{L}}_{2}\right)}, \lambda_{\min \left(\widehat{\mathscr{L}}_{3}\right)}, \lambda_{\min \left(\widehat{\mathscr{L}}_{)}\right)}\right.} \\
& =\frac{3.0003}{0.0913}=32.8620 .
\end{aligned}
$$

Hence, one chooses the coupling strength as $c=35$. Therefore, we can obtain the feedback control gain $c K=$ [-36.8102 -48.1660].

In the next, we are going to illustrate the astringency of multiagent systems under the designed controller. Under four 


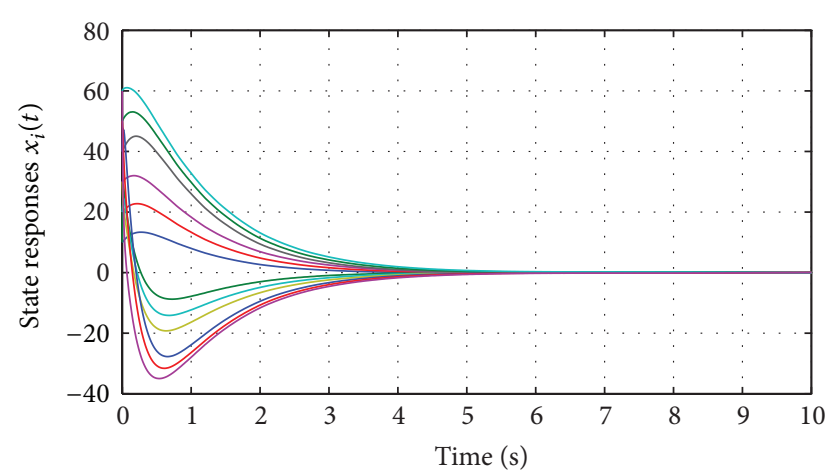

FIgURE 2: The state trajectories of six agents with topology $\mathscr{G}_{1}$.

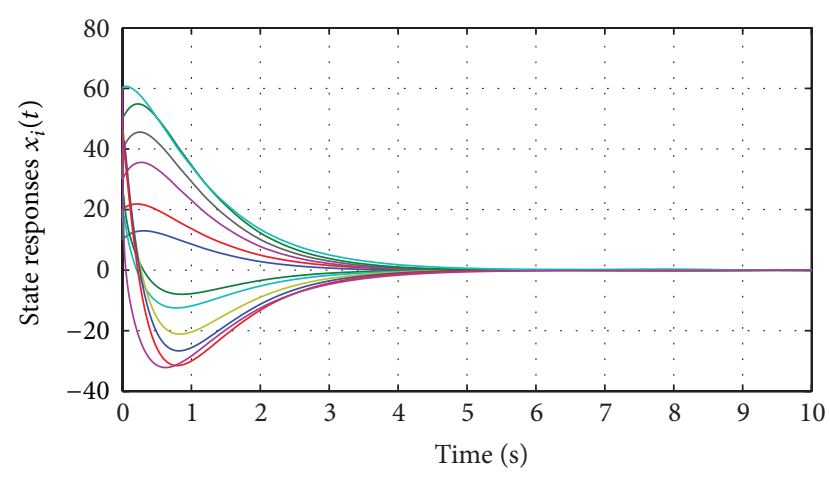

FIgURE 3: The state trajectories of six agents with topology $\mathscr{G}_{2}$.

different graphs, simulation results of six agents with parameter uncertainties will be described. The external disturbances are given by

$$
w_{i}(t)=\left[\begin{array}{l}
\sin (t) \\
\cos (t)
\end{array}\right], \quad i=1, \ldots, 6
$$

The initial conditions of agents are defined as follows:

$$
\begin{array}{lll}
x_{1}(0)=\left[\begin{array}{l}
10 \\
10
\end{array}\right], & x_{2}(0)=\left[\begin{array}{l}
20 \\
20
\end{array}\right], & x_{3}(0)=\left[\begin{array}{l}
30 \\
30
\end{array}\right], \\
x_{4}(0)=\left[\begin{array}{l}
40 \\
40
\end{array}\right], & x_{5}(0)=\left[\begin{array}{l}
50 \\
50
\end{array}\right], & x_{6}(0)=\left[\begin{array}{l}
60 \\
60
\end{array}\right] .
\end{array}
$$

Figures 2, 3, 4, and 5 show the consensus of agents with the designed robust energy-to-peak control protocol and their state trajectories under communication topologies $\mathscr{G}_{1}-\mathscr{G}$, respectively. It can be seen that the state trajectories under four different graphs are almost the same which means that, although topology graphs are altered, the designed controller can still make the multiagent systems with norm bounded uncertainties achieve the consensus. In other words, the designed control protocol is an adaptive one for different cases of communication links.

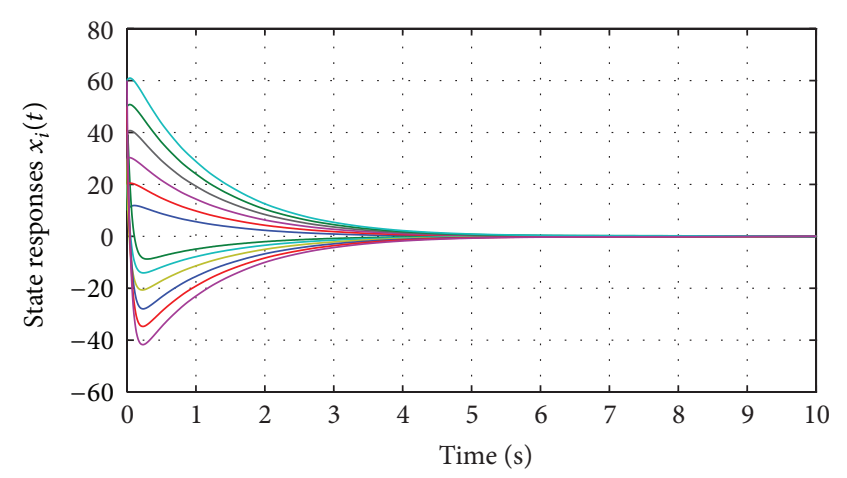

FIgURE 4: The state trajectories of six agents with topology $\mathscr{G}_{3}$.

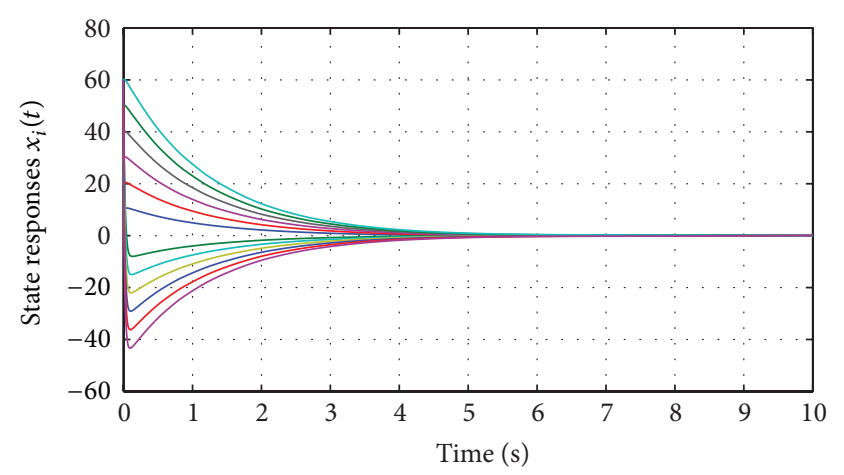

FIGURE 5: The state trajectories of six agents with topology $\mathscr{G}$.

\section{Conclusions}

In this paper, the problem of finite-time distributed $L_{2}-L_{\infty}$ control of uncertain multiagent systems is studied. A cooperative control protocol is described, in which the relative states of agents are used. The finite-time boundedness (FTB) and finite-time $L_{2}-L_{\infty}$ performance of the multiagent systems with parameter uncertainties are both analyzed. Sufficient conditions for the existence of the finite-time $L_{2}-L_{\infty}$ controller are derived in the form of LMIs. Finally, the simulation results show the effectiveness of the proposed approach.

\section{Conflict of Interests}

The authors declare that there is no conflict of interests regarding the publication of the paper.

\section{Acknowledgments}

The authors wish to thank the associate editor and the anonymous reviewers for providing constructive suggestions which have improved the presentation of the paper. This work was supported by National Natural Science Foundation of China (61374213), National Basic Research Program of China (973 Program) (2012CB720000), and the Fundamental Research Funds for the Central Universities (HIT. NSRIF. 2014038). 


\section{References}

[1] W. Ren and R. W. Beard, "Decentralized scheme for spacecraft formation flying via the virtual structure approach," Journal of Guidance, Control, and Dynamics, vol. 27, no. 1, pp. 73-82, 2004.

[2] G. L. Slater, S. M. Byram, and T. W. Williams, "Collision avoidance for satellites in formation flight," Journal of Guidance, Control, and Dynamics, vol. 29, no. 5, pp. 1140-1146, 2006.

[3] W. Ren and N. Sorensen, "Distributed coordination architecture for multi-robot formation control," Robotics and Autonomous Systems, vol. 56, no. 4, pp. 324-333, 2008.

[4] T. Liu and Z. P. Jiang, "Distributed formation control of nonholonomic mobile robots without global position measurements," Automatica, vol. 49, no. 2, pp. 592-600, 2013.

[5] C. Tomlin, G. J. Pappas, and S. Sastry, "Conflict resolution for air traffic management: a study in multiagent hybrid systems," IEEE Transactions on Automatic Control, vol. 43, no. 4, pp. 509$521,1998$.

[6] W. Ren and E. Atkins, "Distributed multi-vehicle coordinated control via local information exchange," International Journal of Robust and Nonlinear Control, vol. 17, no. 10-11, pp. 1002-1033, 2007.

[7] J. Qin, W. X. Zheng, and H. Gao, "Consensus of multiple second-order vehicles with a time-varying reference signal under directed topology," Automatica, vol. 47, no. 9, pp. 19831991, 2011.

[8] Y. Su and J. Huang, "Cooperative output regulation with application to multi-agent consensus under switching network," IEEE Transactions on Systems, Man, and Cybernetics B, vol. 42, no. 3, pp. 864-875, 2012.

[9] P. Lin, Z. Li, Y. Jia, and M. Sun, "High-order multi-agent consensus with dynamically changing topologies and timedelays," IET Control Theory \& Applications, vol. 5, no. 8, pp. 976981, 2011.

[10] Y. Zheng, Y. Zhu, and L. Wang, "Consensus of heterogeneous multi-agent systems," IET Control Theory \& Applications, vol. 5, no. 16, pp. 1881-1888, 2011.

[11] F. Xiao and L. Wang, "Consensus problems for highdimensional multi-agent systems," IET Control Theory \& Applications, vol. 1, no. 3, pp. 830-837, 2007.

[12] Y. Su and J. Huang, "Cooperative global output regulation of heterogeneous second-order nonlinear uncertain multi-agent systems," Automatica, vol. 49, no. 11, pp. 3345-3350, 2013.

[13] L. Ding, Q.-L. Han, and G. Guo, "Network-based leaderfollowing consensus for distributed multi-agent systems," Automatica, vol. 49, no. 7, pp. 2281-2286, 2013.

[14] J. Xu, H. Zhang, and L. Xie, "Input delay margin for consensus ability of multi-agent systems," Automatica, vol. 49, no. 6, pp. 1816-1820, 2013.

[15] B. C. Wang and J. F. Zhang, "Distributed output feedback control of Markov jump multi-agent systems," Automatica, vol. 49, no. 5, pp. 1397-1402, 2013.

[16] H. Zhang, J. Wang, and Y. Shi, "Robust $H_{\infty}$ sliding-mode control for Markovian jump systems subject to intermittent observations and partially known transition probabilities," Systems \& Control Letters, vol. 62, no. 12, pp. 1114-1124, 2013.

[17] H. Zhang, Y. Shi, and M. X. Liu, " $H_{\infty}$ switched filtering for networked systems based on delay occurrence probabilities," ASME Transactions, Journal of Dynamic Systems, Measurement, and Control, vol. 135, no. 6, Article ID 061002, 2013.
[18] H. Zhang, Y. Shi, and M. X. Liu, " $H_{\infty}$ step tracking control for networked discrete-time nonlinear systems with integral and predictive actions," IEEE Transactions on Industrial Informatics, vol. 9, no. 1, pp. 337-345, 2013.

[19] H. Zhang and Y. Shi, "Parameter-dependent $H_{\infty}$ filtering for linear time-varying systems," ASME Transactions, Journal of Dynamic Systems, Measurement, and Control, vol. 135, no. 2, Article ID 021006, 2013.

[20] H. Zhang, Y. Shi, and J. Wang, "On energy-to-peak filtering for nonuniformly sampled nonlinear systems: a Markovian jump system approach," IEEE Transactions on Fuzzy Systems, 1 page, 2013.

[21] H. Zhang, Y. Shi, A. Saadat Mehr, and H. Huang, "Robust FIR equalization for time-varying communication channels with intermittent observations via an LMI approach," Signal Processing, vol. 91, no. 7, pp. 1651-1658, 2011.

[22] H. Zhang, A. S. Mehr, and Y. Shi, "Improved robust energy-topeak filtering for uncertain linear systems," Signal Processing, vol. 90, no. 9, pp. 2667-2675, 2010.

[23] H. Zhang, Y. Shi, and A. Saadat Mehr, "Robust energy-to-peak filtering for networked systems with time-varying delays and randomly missing data," IET Control Theory \& Applications, vol. 4, no. 12, pp. 2921-2936, 2010.

[24] X. Lin, H. Du, S. Li, and Y. Zou, "Finite-time stability and finitetime weighted $L_{2}$-gain analysis for switched systems with timevarying delay," IET Control Theory \& Applications, vol. 7, no. 7, pp. 1058-1069, 2013.

[25] F. Amato, G. Carannante, G. D. Tommasi, and A. Pironti, "Input-output finite-time stability of linear systems: necessary and sufficient conditions," IEEE Transactions on Automatic Control, vol. 57, no. 12, pp. 3051-3063, 2012.

[26] F. Amato, M. Ariola, and C. Cosentino, "Finite-time stability of linear time-varying systems: analysis and controller design," IEEE Transactions on Automatic Control, vol. 55, no. 4, pp. 1003 1008, 2010.

[27] J. Xu and J. Sun, "Finite-time stability of linear time-varying singular impulsive systems," IET Control Theory \& Applications, vol. 4, no. 10, pp. 2239-2244, 2010.

[28] Z. Li, Z. Duan, L. Xie, and X. Liu, "Distributed robust control of linear multi-agent systems with parameter uncertainties," International Journal of Control, vol. 85, no. 8, pp. 1039-1050, 2012.

[29] Z. Li, Z. Duan, and G. Chen, "On $H_{\infty}$ and $H_{2}$ performance regions of multi-agent systems," Automatica, vol. 47, no. 4, pp. 797-803, 2011.

[30] S. He and C. Lin Liu, "Finite-time $H_{\infty}$ fuzzy control of nonlinear jump systems with time delays via dynamic observer-based state feedback," IEEE Transactions on Fuzzy Systems, vol. 20, no. 4, pp. 605-614, 2012.

[31] M. A. Rotea, “The generalized $\mathrm{H}_{2}$ control problem," Automatica, vol. 29, no. 2, pp. 373-385, 1993.

[32] P. Shi, E.-K. Boukas, and R. K. Agarwal, "Control of Markovian jump discrete-time systems with norm bounded uncertainty and unknown delay," IEEE Transactions on Automatic Control, vol. 44, no. 11, pp. 2139-2144, 1999. 


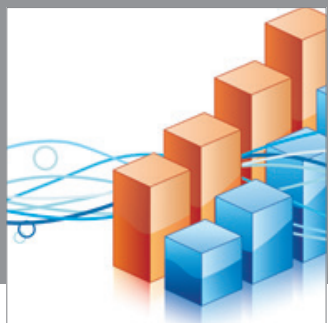

Advances in

Operations Research

mansans

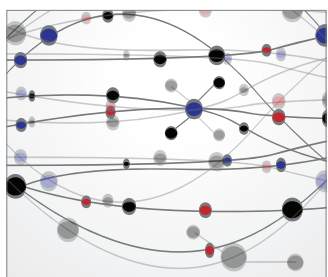

The Scientific World Journal
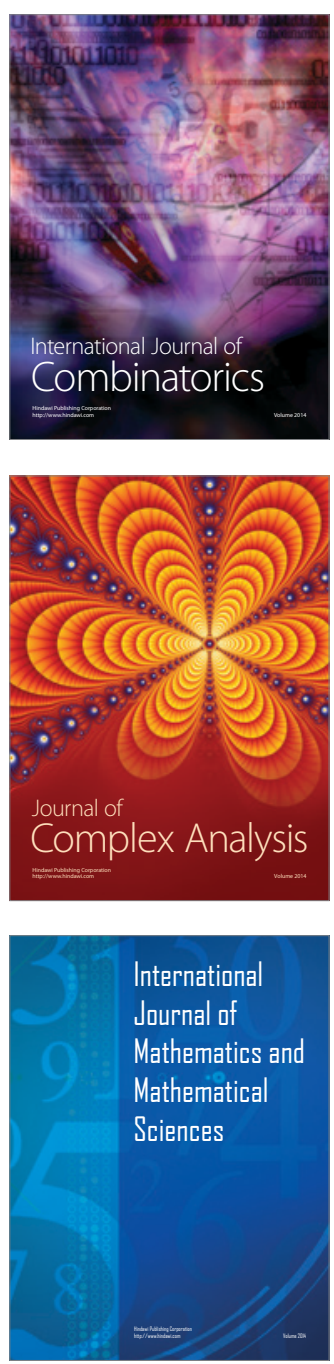
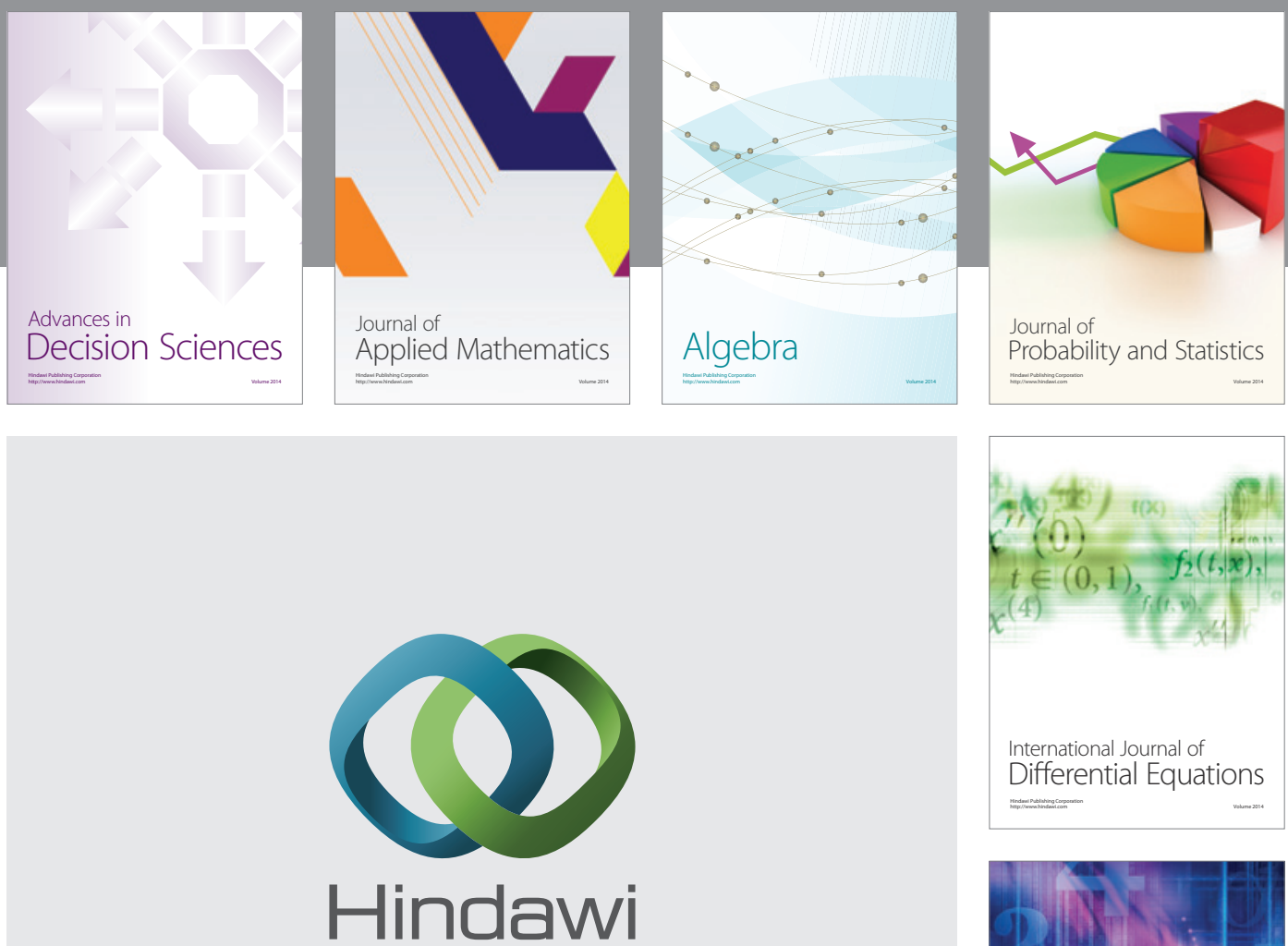

Submit your manuscripts at http://www.hindawi.com
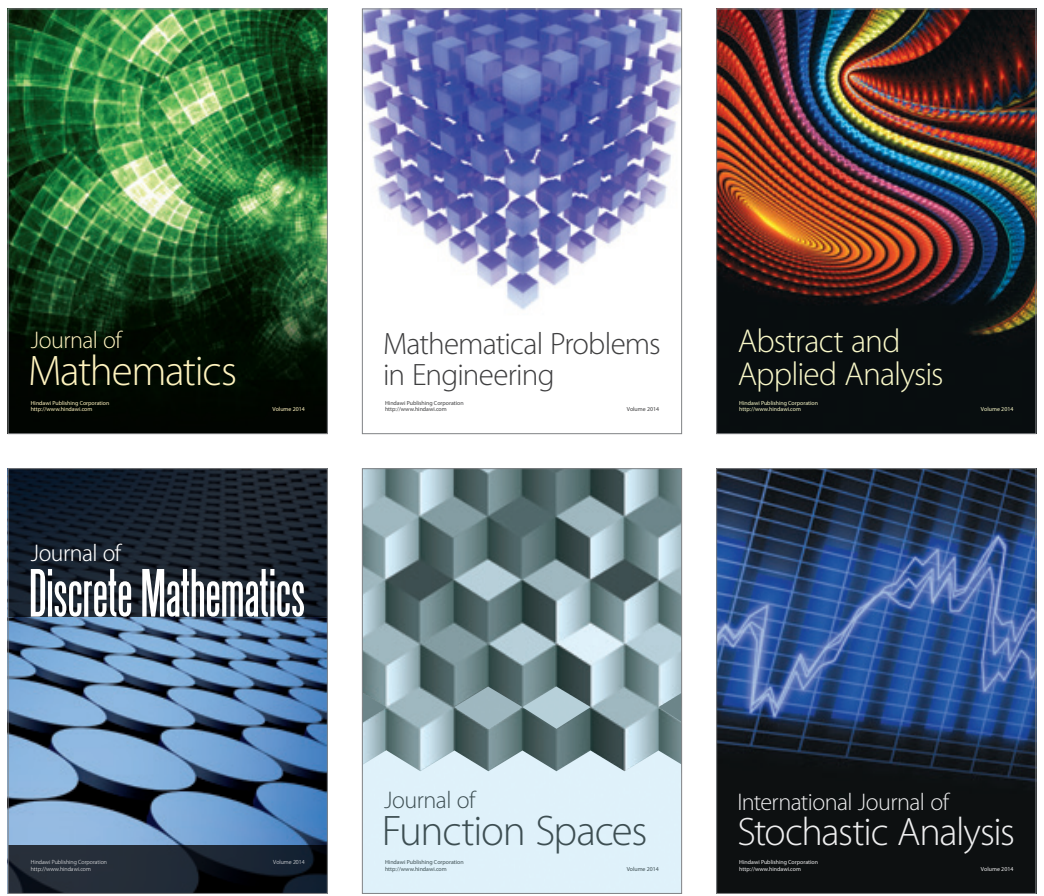

Journal of

Function Spaces

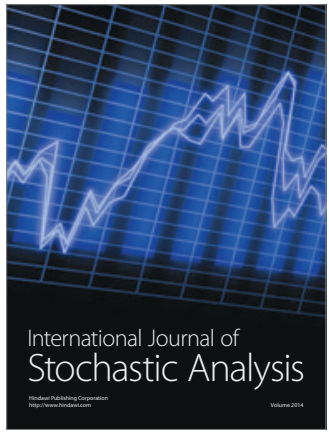

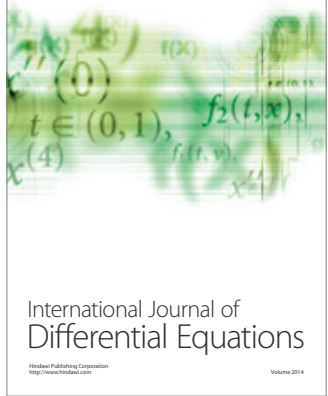
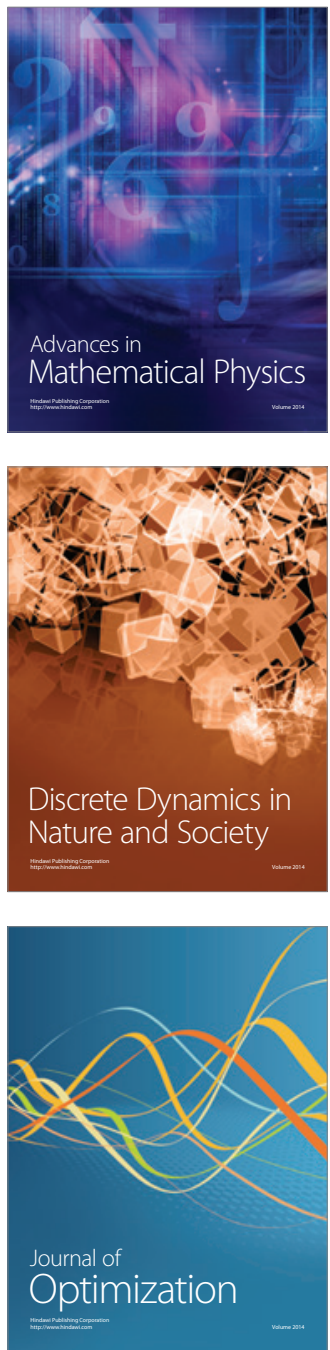